\title{
Characterization of Nanorod Structure Using Spectroscopic Ellipsometry
}

\author{
N. E. J. Omaghali \\ Department of Physics, University of Jos, Jos, Nigeria \\ Email: omaghs@yahoo.com
}

Received 28 January 2016; accepted 18 April 2016; published 21 April 2016

Copyright (C) 2016 by author and Scientific Research Publishing Inc.

This work is licensed under the Creative Commons Attribution International License (CC BY). http://creativecommons.org/licenses/by/4.0/

(c) (i) Open Access

\begin{abstract}
We investigate the resonance modes of gold nanorods on an Indium tin oxide (ITO) coated glass substrate using spectroscopic ellipsometry. The unit cell of the structure investigated is composed of two gold nanorods with differing lengths. In such a structure, we can excite the bright resoance and the dark resonance modes. Numerical simulation of the gold nanorod on substrate was performed with the bright resonance mode at $825.0 \mathrm{~nm}$ and the dark resonance mode at $1107.1 \mathrm{~nm}$ respectively. Using spectroscopic ellipsometry we optically characterize the fabricated gold nanostructure, with the bright resonance mode at $700.0 \mathrm{~nm}$ and the dark resonance mode at $1350.0 \mathrm{~nm}$ respectively. The experimental results from ellipsometry show a good agreement with the results from simulation.
\end{abstract}

\section{Keywords}

Spectroscopy, Ellipsometry, Nanorod, Resonance Modes

\section{Introduction}

The advances in nanofabrication procedures have led to an evolution in the experimental study of nanostructures. The use of techniques such as the electron beam lithography [1]-[5] and nanoimprint lithography [6]-[8] has enabled the fabrication of structures with dimensions in the nanometer range, thereby enhancing further research in nanofabricated structures. In a structure with asymmetry, the dark or subradiant mode is shown to be excited [9]-[13]. In our study, our focus is mainly on the optical characterization of the fabricated structure. With advances in nano fabrication techniques also comes with it improved methods for optical characterization of the fabricated nanostructures. A common technique for the optical characterization of nanostructures involves normal incidence transmission and reflection measurements [14].

Another technique which can be employed is the method of ellipsometry, a method dependent on the changes 
in amplitude and phase of polarized light due to their interaction with matter. Measurements with ellipsometer are non contact thereby making it a suitable tool for the investigation of nanostructures. In ellipsometry the changes in the polarization state of light after reflection on a sample or after transmission through a sample can be measured and from these different properties of materials can be established. The ellipsometer is able to measure the amplitude ratio and also the phase difference between the p-polarized and the s-polarized light. The ellipsometer offers the added advantage of obtaining phase information, thereby making it a powerful optical characterization technique [15] [16]. Different nanostructures such as split ring resonators [7], fishnet metamaterial [17] [18] etc. have been characterized using the method of ellipsometry. The method of ellipsometry has also been shown to be very useful in determining the thickness and properties of thin films [19] [20]. In this work, I employ the method of spectroscopic ellipsometry to optically characterize a fabricated gold nanorod structure. The structure whose unit cell is composed of two gold nanorods of unequal lengths is simulated and the bright and dark resonances for electromagnetic wave incident normally on the structure are obtained. Using the method of ellipsometer we then optically characterized the fabricated gold nanorod on glass substrate. Our experimental results from ellipsometry are found to be in agreement with the results from simulation. Also, aside from the identifying the resonances from the ellipsometric experimental measurements, by using the method of ellipsometry we have obtained the phase information of the fabricated structure.

\section{Design and Fabrication}

The gold rod on substrate under investigation is presented here. The structure is composed of gold nanorods deposited on an Indium Tin Oxide (ITO) coated glass substrate. A schematic of the unit cell of the structure is shown in Figure 1, which is composed of two gold nanorod of unequal lengths deposited on a substrate. The lengths of the gold nanorods are L1 and L2 respectively. The gold nanorods L1 and L2 are separated by a distance $d$ of 50nm as shown in Figure 1. One of the gold nanorods of the unit cell has a length L1 of $170 \mathrm{~nm}$ while the other gold nanorod of the unit cell has a length L2 of $200 \mathrm{~nm}$ respectively. The gold nanorods L1 and L2 both have equal width $w$ of $70 \mathrm{~nm}$ each and an equal thickness of $30 \mathrm{~nm}$. The unit cell is such that its periodicity is $300 \mathrm{~nm}$ along the $\mathrm{x}$ direction and $300 \mathrm{~nm}$ along y direction respectively. The index of the glass substrate is taken to be 1.5. The permittivity of the ITO is 3.8 while its thickness is $25 \mathrm{~nm}$.

We performed the electromagnetic simulation of the gold nanorod on ITO coated glass substrate structure of Figure 1 using finite difference time domain (FDTD). With the incident wave normal to the plane containing the gold nanorods of Figure 1, and with the electric field component along the length of the gold nanorods or x-direction, we obtain the transmission spectrum for the gold nanorod on ITO coated glass substrate. The transmission spectrum from simulation for the structure of Figure 1 is depicted in Figure 2. From the transmission spectrum, two resonance dips can be observed. The first resonance dip is at the shorter wavelength of $825.0 \mathrm{~nm}$ and has a broad profile. The second resonance dip is at the longer wavelength of $1107.1 \mathrm{~nm}$ and has a narrow profile compared to the first resonance. The first resonance at $825.0 \mathrm{~nm}$ is bright resonant mode with symmetric electric field distribution while the second resonance at $1107.1 \mathrm{~nm}$ is a dark resonant mode with a characteristic

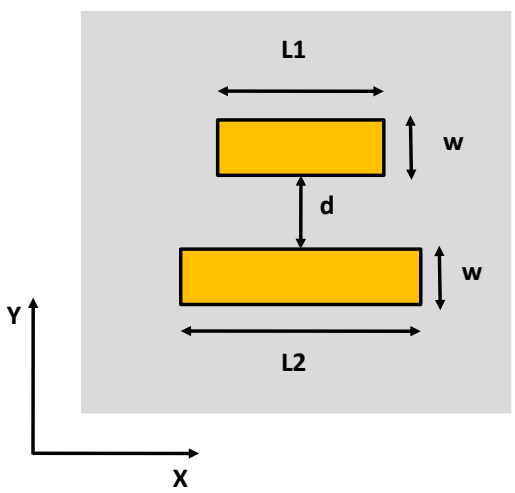

Figure 1. Gold nanorods on an Indium tin oxide (ITO) coated glass substrate. The gold has a thickness of $30 \mathrm{~nm}$ with lengths L1 of 170 $\mathrm{nm}$ and L2 of $200 \mathrm{~nm}$ respectively. The gold nanorods have equal widths of $70 \mathrm{~nm}$. 


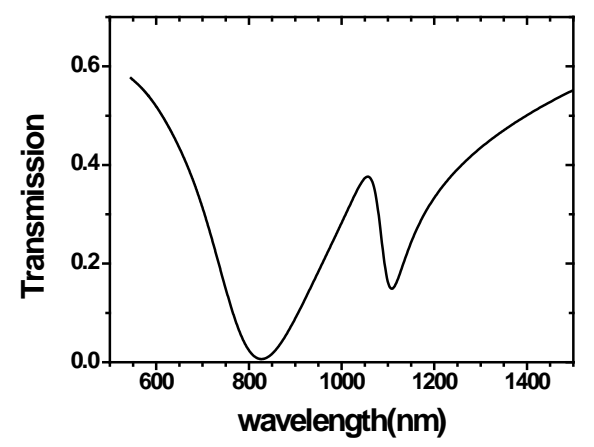

Figure 2. Transmission spectra versus wavelength for the gold nanorod on ITO coated glass substrate with resonances at $825.0 \mathrm{~nm}$ and $1107.1 \mathrm{~nm}$.

anti-symmetric electric field distribution [9]-[13]. It is worth mentioning that for the case where the length L1 is equal to L2 in Figure 1, only the shorter wavelength resonance having a broad spectrum is obtained (not shown). However, for lengths L1 not equal to L2 we can excite the dark resonance at the longer wavelength as shown in Figure 2.

The gold nanorod on ITO coated glass substrate shown in Figure 1 was fabricated and the basic fabrication processes are outlined. After cleaning the ITO-coated glass substrate it was spin coated with polymethyl metacrylate (PMMA) which is a positive photoresist [21]. Afterwards, the sample is then exposed to electron beam lithography (EBL) for the nano structure pattern writing on the PMMA with an exposure dose of $500 \mathrm{~mJ} / \mathrm{cm}^{2}$. After the pattern writing, the sample is then developed using a developer solution of methyl isobutyl ketonebonds (MIBK). Following this, the sample is then rinsed in a solution of Isopropyl alcohol (IPA) and then dried using nitrogen flow. A scanning electron microscope (SEM) image of the fabricated structure of Figure 1 is shown Figure 3.

\section{Optical Characterization}

The optical characterization of the fabricated gold nanorod on ITO coated glass substrate (Figure 3), was carried out using the Variable Angle Spectroscopic Ellipsometer (VASE). Using the method of ellipsometry, the polarization ratios for oblique incident reflected and transmitted beams can be obtained. Ellipsometry uses the ratio of polarized reflection coefficients $\rho$ which can be expressed in terms of the ellipsometric angles $\Psi$ and $\Delta$, given by

$$
\rho=\frac{R_{p}}{R_{s}}=\tan \Psi \mathrm{e}^{i \Delta}
$$

where $\Psi$ is the amplitude ratio of reflection coefficient and $\Delta$ is the phase difference of the reflection coefficient for the $\mathrm{p}$ and s directions respectively [7] [15] [16]. $R_{p}$ is the complex-valued reflection coefficient for light polarized parallel (p-polarized) to the plane of incidence and $R_{s}$ is the complex-valued reflection coefficient for light polarized perpendicular (s-polarized) to the plane of incidence [15]. The fabricated sample shown in Figure 3 is characterized under variable angle spectroscopic ellipsometry (VASE) for angles of incidence (AOI) ranging from $0^{\circ}$ to $35^{\circ}$ at increments of $5^{\circ}$. In our measurements, the incidence plane is the $\mathrm{xz}$ plane of Figure 1 and the electric field is along the length of the gold nanorod or along the x-axis as shown in Figure 1. The measured representative VASE data ( $\Psi$ and $\Delta$ ) of the ellipsometric angles for the sample for angles of incidence (AOI) from $0^{\circ}$ to $35^{\circ}$ are shown in Figure 4 and Figure 5 respectively.

\section{Results and Discussion}

The VASE $\Psi$ spectrum in Figure 4 is the p-polarized spectra of the fabricated nanostructure (Figure 4) in transmission, which depicts the measured $\Psi$ versus the wavelength. As can be seen from Figure 4, a first resonance dip is observed at the shorter wavelength of about $700 \mathrm{~nm}$ and a second dip observed at the longer wavelength of about $1350 \mathrm{~nm}$. The first dip at about $700 \mathrm{~nm}$ corresponds to the bright resonance mode. This resonant 


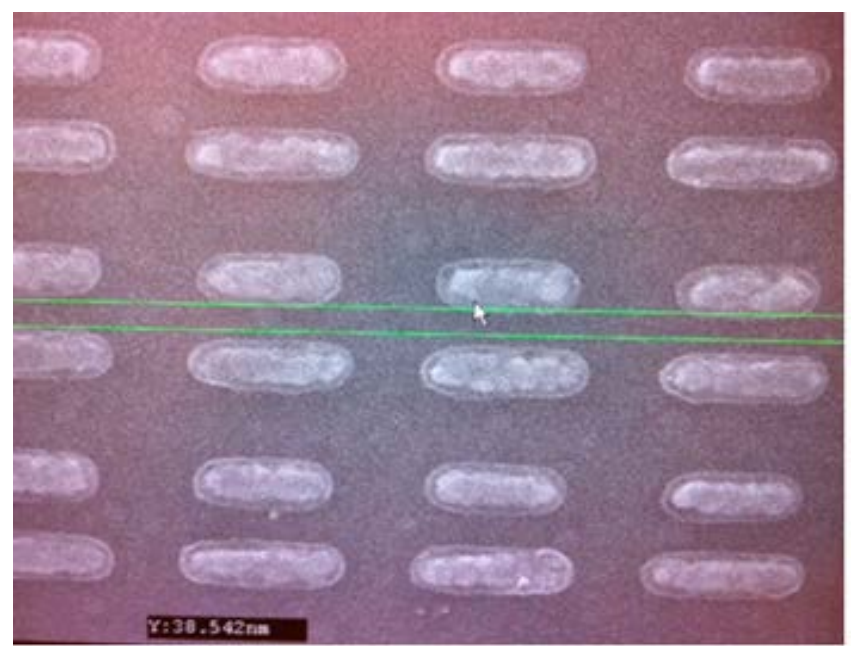

Figure 3. Scanning electron microscope (SEM) image of the fabricated gold nanorods on an Indium tin oxide (ITO) coated glass substrate.

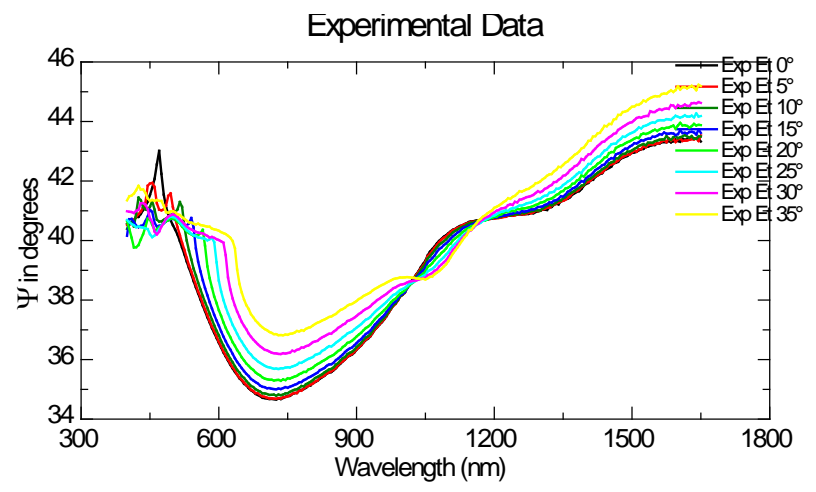

Figure 4. Representative VASE $\Psi$ spectra for the transmission ellipsometry of the gold nanostructure. $\Psi$ in degrees versus wavelength in nanometers for angles of incidence (AOI) from $0^{\circ}$ to $35^{\circ}$ at intervals of $5^{\circ}$. The incidence plane is perpendicular to the gold nanorods (p-polarization).

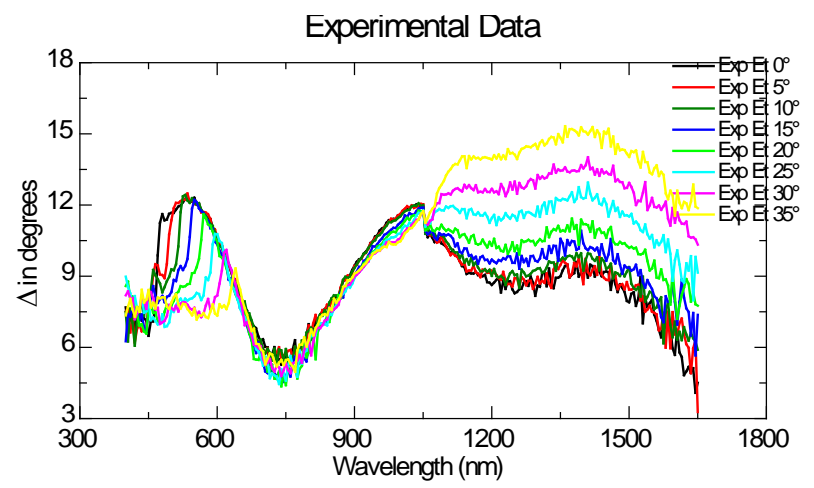

Figure 5. Representative VASE $\Delta$ spectra for the transmission ellipsometry of the gold nanostructure. The $\Delta$ in degrees versus wavelength in nanometers for angles of incidence from $0^{\circ}$ to $35^{\circ}$. The incidence plane is perpendicular to the gold nanorods (p-polarization). 
mode at about $700 \mathrm{~nm}$ is the electric resonance with a characteristic broad resonance profile, having low quality factor owing to the low loss nature of the mode. These dips correspond to the resonance modes of the gold nanostructure as depicted in Figure 2.

In Figure 4 of the representative VASE $\Psi$ spectra, a second dip can be observed at the longer wavelength of $1350 \mathrm{~nm}$. This second resonance mode at the longer wavelength of about $1350 \mathrm{~nm}$ has a characteristic narrow profile and consequently a high quality factor, unlike the first resonance at $700 \mathrm{~nm}$.

Likewise, Figure 5 shows the measured VASE $\Delta$ spectra for the gold nanostructure (Figure 3) against wavelength for incidence angles (AOI) from $0^{\circ}$ to $35^{\circ}$, at intervals of $5^{\circ}$. From the experimental measurement (Figure 5), a first resonance at the shorter wavelength of about $700 \mathrm{~nm}$ is observed, which corresponds to the resonance mode observed from the VASE $\Psi$ spectra of Figure 4. Also from the VASE $\Delta$ spectra of Figure 4, a second resonance mode at the longer wavelength of about $1350 \mathrm{~nm}$ is observed, similar to the observed second resonance in the VASE $\Psi$ spectra of Figure 5, consistent with experimental results. It is worth noting that the variance of the resonance position in Figure 2 with the experimentally obtained spectrum of Figure 4 and Figure 5 can be attributed to imperfections in the fabrication procedures.

\section{Conclusion}

In this work the spectroscopic ellipsometry has been used in investigating a gold nanorod on an ITO coated glass substrate. The optical spectrum of the fabricated gold nanorod was experimentally obtained using ellipsometry. From the experimental results in Figure 4 and Figure 5 the observed resonance of the gold nanorod is in agreement with that from the simulated results in Figure 2. The discrepancy in the positions of resonance of the experimental measurements and simulation results is attributed to fabrication imperfections. The sensitive nature of ellipsometry measurements to polarization changes has enabled the clear identification of the resonant modes of the fabricated gold nanorod on Indium tin oxide (ITO) coated glass substrate.

\section{References}

[1] Taylor, A.B., Michaux, P., Mohsin, A.S. and Chon, J.W. (2013) Electron-Beam Lithography of Plasmonic Nanorod Arrays for Multilayered Optical Storage. Optics Letters, 38, 3969-3972.

[2] Taylor, A.B., Michaux, P., Mohsin, A.S.M. and Chon, J.W.M. (2014) Electron-Beam Lithography of Plasmonic Nanorod Arrays for Multilayered Optical Storage. Optics Express, 22, 13234-13243. http://dx.doi.org/10.1364/OE.22.013234

[3] Cinel, N.A., Bütün, S. and Özbay, E. (2012) Electron Beam Lithography Designed Silver Nano-Disks Used as Label Free Nano-Biosensors Based on Localized Surface Plasmon Resonance. Optics Express, 20, 2587-2597. http://dx.doi.org/10.1364/OE.20.002587

[4] DeRose, G.A., Zhu, L., Poon, J.K.S., Yariv, A. and Scherer, A. (2007) Electron-Beam Lithography Techniques for Micro and Nano-Scale Surface Structure Current Injection Lasers. Conference on Lasers and Electro-Optics (CLEO), Baltimore, 6-11 May 2007, 1-2. http://dx.doi.org/10.1109/cleo.2007.4452835

[5] Vieu, C., Carcenac, F., Pépin, A., Chen, Y., Mejias, M., Lebib, A., Manin-Ferlazzo, L., Couraud, L. and Launois, H. (2000) Electron Beam Lithography: Resolution Limits and Applications. Applied Surface Science, 164, 111-117. http://dx.doi.org/10.1016/S0169-4332(00)00352-4

[6] Bergmair, I., Dastmalchi, B., Bergmair, M., Saeed, A., Hilber, W., Hesser, G., Helgert, C., Pshenay-Severin, E., Pertsch, T., Kley, E.B., Hübner, U., Shen, N.H., Penciu, R., Kafesaki, M., Soukoulis, C.M., Hingerl, K., Muehlberger, M. and Schoeftner, R. (2011) Single and Multilayer Metamaterials Fabricated by Nanoimprint Lithography. Nanotechnology, 22, Article ID: 325301.

[7] Oates, T.W.H., Wormeester, H. and Arwin, H. (2011) Characterization of Plasmonic Effects in Thin Films and Metamaterials Using Spectroscopic Ellipsometry. Progress in Surface Science, 86, 328-376. http://dx.doi.org/10.1016/j.progsurf.2011.08.004

[8] Ahn, S.H. and Guo, L.J., (2009) Large-Area Roll-to-Roll and Roll-to-Plate Nanoimprint Lithography: A Step toward High-Throughput Application of Continuous Nanoimprinting. ACS Nano, 3, 2304-2310. http://dx.doi.org/10.1021/nn9003633

[9] Jeyaram, T., Verellen, N., Zheng, X., Silhanek, A.V., Hojeiji, M., Terhalle, B., Ekinci, Y., Valev, V.K., Vandenbosch, G.A.E. and Moshchalkov, V.V. (2013) Rendering Dark Modes Bright by Using Asymmetric Split Ring Resonators. Optics Express, 21, 15464-15474. http://dx.doi.org/10.1364/OE.21.015464 
[10] Cao, W., Singh, R.., Al-Naib, I.A.I., He, M., Taylor, A.J. and Zhang, W. (2012) Low-Loss Ultra-High-Q Dark Mode Plasmonic Fano Metamaterials. Optics Letters, 37, 3366-3368. http://dx.doi.org/10.1364/OL.37.003366

[11] Papasimakis, N., Fu, Y.H., Fedotov, F.A., Prosvirnin, S.L., Tsai, D.P. and Zheludev, N.I. (2009) Metamaterial with Polarization and Direction Insensitive Resonant Transmission Response Mimicking Electromagnetically Induced Transparency. Applied Physics Letters, 94, 211902-211904. http://dx.doi.org/10.1063/1.3138868

[12] Singh, R., Al-Naib, I.A.I., Koch, M. and Zhang, W. (2011) Sharp Fano Resonances in THz Metamaterials. Optics Express 19, 6314-6319. http://dx.doi.org/10.1364/oe.19.006312

[13] Christ, A., Martin, O.J.F., Ekinci, Y., Gippius, N.A. and Tikhodeev, S.G. (2008) Symmetry Breaking in a Plasmonic Metamaterial at Optical Wavelength. Nano Letters, 8, 2171-2175. http://dx.doi.org/10.1021/nl0805559

[14] Smith, D.R., Schultz, S., Markos, P. and Soukoulis, C.M. (2002) Determination of Effective Permittivity and Permeability of Metamaterials from Reflection and Transmission Coefficients. Physical Review B, 65, 195104. http://dx.doi.org/10.1103/PhysRevB.65.195104

[15] Fujiwara, H. (2003) Spectroscopic Ellipsometry: Principles and Applications. John Wiley \& Sons Ltd., Chichester.

[16] Tompkins, H.G. and Irene, E.A. (2005) Handbook of Ellipsometry. William Andrew, New York. http://dx.doi.org/10.1007/3-540-27488-X

[17] Oates, T.W.H., Dastmalchi, B., Isic, G., Tollabimazraehno, S., Helgert, C., Pertsch, T., Kley, E., Verschuuren, M.A., Bergmair, I., Hingerl, K. and Hinrichs, K. (2012) Oblique Incidence Ellipsometric Characterization and the Substrate Dependence of Visible Frequency Fishnet Metamaterials. Optics Express, 20, 11166-11177. http://dx.doi.org/10.1364/OE.20.011166

[18] Khosroabadi, A.A., Gangopadhyay, P., Cocilovo, B., Makai, L., Basa, P., Duong, B., Thomas, J. and Norwood, R.A. (2013) Spectroscopic Ellipsometry on Metal and Metal-Oxide Multilayer Hybrid Plasmonic Nanostructures. Optics Express, 38, 3969-3972. http://dx.doi.org/10.1364/ol.38.003969

[19] Toudert, J. (2013) Spectroscopic Ellipsometry for Active Nano and Metamaterials. Nanotechnology Reviews, 3, 223245.

[20] Gong, J., Dai, R., Wang, Z. and Zhang, Z. (2015) Thickness Dispersion of Surface Plasmon of Ag Nano-Thin Films: Determination by Ellipsometry Iterated with Transmittance Method. Scientific Reports, 5, Article Number: 9279. http://dx.doi.org/10.1038/srep09279

[21] Chou, S.Y., Krauss, P.R. and Renstrom, P.J. (1996) Imprint Lithography with 25-Nanometer Resolution. Science, 272, 85-87. http://dx.doi.org/10.1126/science.272.5258.85 